\title{
DIFFERENTIAL INVARIANTS OF A PLANE AND OF CURVES IN THE PLANE.
}

\author{
By A. R. Forsyth (Cambridge, Engl.).
}

Adunanza del 23 luglio 1905.

I. In a couple of memoirs published elsewhere ${ }^{*}$ ), I have discussed the differential invariants, (i) of a surface and of curves upon the surface, and (ii) of ordinary space and of surfaces and curves in that space. The method adopted is an application of LIE's theory of continuous groups and is a modification of a development ${ }^{* *}$ ) due to Professor Zorawski. The object of the present note is to illustrate the method, by using it to obtain the differential invariants of a plane and of curves in the plane.

The coordinates of a point are denoted by $x$ and $y$; and we take two independent variables $u$ and $v$, being functions of $x$ and $y$. Conversely, $x$ and $y$ are two independent functions of $u$ and $v$. It is not assumed that the curves $u=$ constant, $v=$ constant, are orthogonal to one another.

The only fundamental magnitudes that are intrinsic to the plane are $a, b, b$, where

$$
\begin{gathered}
a=\left(\frac{\partial x}{\partial u}\right)^{2}+\left(\frac{\partial y}{\partial u}\right)^{2}, \quad b=\left(\frac{\partial x}{\partial v}\right)^{2}+\left(\frac{\partial y}{\partial v}\right)^{2}, \\
b=\frac{\partial x}{\partial u} \frac{\partial x}{\partial v}+\frac{\partial y}{\partial u} \frac{\partial y}{\partial v} ;
\end{gathered}
$$

evidently the element of arc in the plane is given by

We also have

$$
d s^{2}=d x^{2}+d y^{2}=a d u^{2}+2 b d u d v+b d v^{2} .
$$

$$
a b-b^{2}=\left[\frac{\partial(x, y)}{\partial(u, v)}\right]^{2}=J^{2}
$$

using $J$ to denote the Jacobian of $x$ and $y$.

We are to be concerned with curves in the plane: any such curve will be denoted by

$$
\varphi(u, v)=\text { constant }
$$

where the value of the constant is immaterial. 
2. A quantity $f$ is defined to be a differential invariant when, if the same function $f^{\prime}$ of new independent variables $u^{\prime}$ and $v^{\prime}$ is formed, the relation

$$
f=\Omega^{\mu} f^{\prime}
$$

is satisfied identically, where (for our purposes) $\mu$. is a positive whole number including zero, and where

$$
\Omega=\frac{\partial\left(u^{\prime}, v^{\prime}\right)}{\partial(u, v)} .
$$

When $\mu$ is different from zero, $f$ is a relative invariant; when $\mu$ is zero, $f$ is an absolute invariant. Obviously $J$ is a relative invariant: we have

$$
J=\Omega J^{\prime} \text {. }
$$

The arguments that can occur in a differential invariant are, firstly, $a, b, b$, and their derivatives with regard to $u$ and $v$ up to any order, say $p-\mathrm{r}$; and, secondly, the derivatives of $\varphi$ with regard to $u$ and $v$ (but not $\varphi$ itself) up to order $p$. In order to express the derivatives of any quantity $\Theta$, we write

$$
\Theta_{\mu \nu}=\frac{\partial^{\mu+\nu} \Theta}{\partial u^{\mu} \partial v^{\nu}},
$$

for all values of $\mu$ and $v$.

3. The process of obtaining invariants is as follows. By a theorem due to LIE, we know that the infinitesimal transformations of a continuous group suffice to determine the group, and therefore we take account only of the increments of the arguments due to these infinitesimal variations. We take these variations in the form

$$
u^{\prime}=u+\xi d t, \quad v^{\prime}=v+n d t
$$

where $\xi$ and $n$ are independent arbitrary functions of $u$ and $v$, and $d t$ is an arbitrary infinitesimal that does not involve $u$ or $v$ : and then, retaining only the first powers of $d t$ as usual, we have

$$
\Omega=\mathrm{I}+\left(\xi_{\mathrm{so}}+n_{\mathrm{O} \mathrm{I}}\right) d t .
$$

Proceeding to obtain the increments of the quantities that can occur in a differential invariant, we find the following:

$$
\begin{aligned}
-\frac{d a_{m n}}{d t} & =\sum \sum\left(\begin{array}{c}
m \\
r
\end{array}\right)\left(\begin{array}{c}
n \\
s
\end{array}\right)\left[a_{m+1-r, n-s} \xi_{r s}+a_{m-r, n+1-s} n_{r s}\right] \\
& +2 \sum \sum\left(\begin{array}{c}
m \\
r
\end{array}\right)\left(\begin{array}{c}
n \\
s
\end{array}\right)\left[a_{m-r, n-s} \xi_{r+1, s}+b_{m-r, n-s} n_{r+1, s}\right] \\
-\frac{d h_{m n}}{d t} & =\sum \sum\left(\begin{array}{c}
m \\
r
\end{array}\right)\left(\begin{array}{c}
n \\
s
\end{array}\right)\left[b_{m+1-r, n-s} \xi_{r s}+b_{m-r, n+1-s} n_{r s}\right] \\
& +\sum \sum\left(\begin{array}{c}
m \\
r
\end{array}\right)\left(\begin{array}{c}
n \\
s
\end{array}\right)\left[a_{m-r, n-s} \xi_{r, s+1}+b_{m-r, n-s} n_{r+1, s}+b_{m-r, n-s}\left(\xi_{r+1, s}+n_{r, s+1}\right)\right] \\
-\frac{d b_{m n}}{d t} & =\sum \sum\left(\begin{array}{c}
m \\
r
\end{array}\right)\left(\begin{array}{c}
n \\
s
\end{array}\right)\left[b_{m+1-r, n-s} \xi_{r s}+b_{m-r, n+1-s} n_{r s}\right] \\
& +2 \sum \sum\left(\begin{array}{c}
m \\
r
\end{array}\right)\left(\begin{array}{c}
n \\
s
\end{array}\right)\left[b_{m-r, n-s} \xi_{r, s+1}+b_{m-r, n-s} n_{r, s+1}\right] \\
-\frac{d \varphi_{m n}}{d t} & =\sum \sum\left(\begin{array}{c}
m \\
r
\end{array}\right)\left(\begin{array}{c}
n \\
s
\end{array}\right)\left[\varphi_{m+1-r, n-s} \xi_{r, s+1}+\varphi_{m-r, n+s-s} n_{r, s+1}\right]
\end{aligned}
$$


holding for all values of $m$ and $n$. The summation $\Sigma \Sigma^{\prime}$ is for all values of $r$ from $o$ to $m$ and of $s$ from o to $n$, excluding simultaneous zero values; the summation $\Sigma \Sigma$ is for the same values of $r$ and of $s$, including simultaneous zero values.

4. These increments are substituted in the equation

$$
f=\Omega^{\mu} f^{\prime}
$$

which defines the invariants: the quantities are expanded in powers of $d t$, and only the first power of $d t$ is retained, so that

$$
\begin{aligned}
f & =\left[\mathrm{I}+\mu\left(\xi_{\mathrm{ro}}+\eta_{\mathrm{o}}\right) d t\right]\left[f+\sum \frac{\partial f}{\partial z} \frac{\partial z}{\partial t} d t\right] \\
& =f+\mu\left(\xi_{10}+n_{01}\right) f d t+\sum \frac{\partial f}{\partial z} \frac{d z}{d t} d t,
\end{aligned}
$$

where the summation extends over all the arguments $z$ that occur in $f$. This equation is to be satisfied for all values of the arbitrary quantities $\dot{\xi}$ and $n$; and therefore the aggregate coefficients of each of the derivatives of these two quantities must vanish. We thus obtain a series of partial differential equations. Two of these have the form

$$
\begin{aligned}
& \mu \cdot f=\sum \sum\left[(m+2) a_{m n} \frac{\partial f}{\partial a_{m n}}+(m+1) b_{m n} \frac{\partial f}{\partial b_{m n}}+m b_{m n} \frac{\partial f}{\partial b_{m n}}+m \varphi_{m n} \frac{\partial f}{\partial \varphi_{m n}}\right], \\
& \mu \cdot f=\sum \sum\left[n a_{m n} \frac{\partial f}{\partial a_{m n}}+(n+1) b_{m n} \frac{\partial f}{\partial b_{m n}}+(n+2) b_{m n} \frac{\partial f}{\partial b_{m n}}+n \varphi_{m n} \frac{\partial f}{\partial \varphi_{m n}}\right],
\end{aligned}
$$

where the summation is for all values of $m$ and of $n$, including simultaneous zeros; and the rest are

$$
\left\{\begin{array}{l}
0=\sum \frac{\partial f}{\partial z} \frac{\partial}{\partial \xi_{r s}}\left(\frac{d z}{d t}\right) \\
0=\sum \frac{\partial f}{\partial z} \frac{\partial}{\partial n_{r s}}\left(\frac{d z}{d t}\right)
\end{array}\right.
$$

for all values of $r$ and $s$ except $r, s=0,0 ; \mathrm{I}, 0 ; 0, \mathrm{I}$. The first two of these equations we replace by the equivalent equations

$$
\left\{\begin{array}{r}
o=\sum \sum\left[(m-n+2) a_{m n} \frac{\partial f}{\partial a_{m n}}+(m-n) b_{m n} \frac{\partial f}{\partial h_{m n}}+(m-n-2) b_{m n} \frac{\partial f}{\partial b_{m n}}\right. \\
\left.+(m-n) \varphi_{m n} \frac{\partial f}{\partial \varphi_{m n}}\right],
\end{array}\right.
$$

(III) $2 \mu f=\sum \sum\left[(m+n+2)\left(a_{m n} \frac{\partial f}{\partial a_{m n}}+b_{m n} \frac{\partial t}{\partial h_{m n}}+b_{m n} \frac{\partial f}{\partial b_{m n}}\right)+(m+n) \varphi_{m n} \frac{\partial f}{\partial \varphi_{m n}}\right]$.

From the general theory of continuous groups we know that any function $f$, which satisfies these equations, possesses the property represented by the definition

$$
f=\Omega^{\mu} f^{\prime}
$$

The equations, included in the aggregate (I) and (II), are homogeneous and linear in the derivatives of $f$; and they are a complete Jacobian system. The equation (III) 
is a quasi-homogeneous equation; when an integral of (I) and (II) of appropriate type is chosen, the equation (III) determines the value of $\mu$. to be associated with that integral.

5. To render the equations more precise, let it be required to obtain the number of invariants, algebraically independent of one another, which involve derivatives of up to otder $p$ and derivatives of $a, b, b$, up to order $p-\mathrm{r}$. The number of derivatives of $\varphi$ (excluding $\varphi$ itself) which can occur is

$$
\begin{aligned}
& 2+3+\cdots+(p+1) \\
= & \frac{1}{2} p(p+3) ;
\end{aligned}
$$

the number of derivatives of $a, b, b$ (including $a, b, b$, themselves) which can occur is

$$
\begin{aligned}
& 3(\mathrm{I}+2+\cdots+p) \\
= & \frac{3}{2} p(p+\mathrm{I}) ;
\end{aligned}
$$

and therefore the total number of arguments which can occur in the invariant $f$ is

$$
\begin{aligned}
& \frac{1}{2} p(p+3)+\frac{3}{2} p(p+\mathrm{I}) \\
= & 2 p^{2}+3 p .
\end{aligned}
$$

The total number of equations in the complete Jacobian system made up of (I) and (II) is less, by unity, than the total number of derivatives of $\xi$ and $n$ (excluding $\xi^{\circ}$ and $n$ themselves) up to order $p$ : hence it is

Consequently, there are

$$
\begin{aligned}
& 2[2+3+\cdots+(p+\mathrm{I})]-\mathrm{I} \\
= & p(p+3)-\mathrm{I} .
\end{aligned}
$$

that is,

$$
2 p^{2}+3 p-[p(p+3)-\mathrm{I}]
$$

$$
p^{2}+I
$$

invariants algebraically independent of one another. Each of these has its appropriate value of $\mu$; and $J$ is one of them, its value of $\mu$ being unity. Hence

$$
f J^{-\mu}
$$

is an absolute invariant; and therefore there are $p^{2}$ absolute invariants which involve derivatives of $q$ up to order $p$ and are algebraically independent of one another.

6. We next have to consider what are the geometrical quantities available for the expression of absolute invariants. Dealing first with only a single curve

$$
\varphi(u, v)=\text { constant, }
$$

let $d n$ denote an element of direction along the normal at any point, and write

$$
\frac{d \varphi}{d n}=C
$$

Then the simplest geometrical quantities of an invariantive kind are $C$ and $\rho$, the radius of curvature: in addition to these, there will be their derivatives. We shall assume these derivatives taken along the tangent and along the normal. 
Now $C$ is a diffcrential quantity of the first order, and $p$ is a differential quantity of the second order; in connection with the preceding absolute invariants, we take derivatives of $C$ of all orders up to $p-\mathrm{I}$, and derivatives of $p$ of all orders up to $p-2$; and the total number of quantities thus arailable is

$$
\begin{aligned}
& {[\mathrm{I}+\cdots+p]+[\mathrm{I}+\cdots+(p-\mathrm{I})] } \\
= & \frac{1}{2} p(p+\mathrm{I})+\frac{1}{2} p(p-\mathrm{I}) \\
= & p^{2}
\end{aligned}
$$

being exactly the same in number as the aggregate of algebraically independent absolute invariants up to the same order. Hence all the geometrical quantities are independent of one another: and therefore there are no relations among the geometrical magnitude among the quantities $C, \rho$, and their derivatives, common to all curves in the plane.

This result differs from the geometry of curves upon a curved surface and from the geometry of surfaces in space ${ }^{*}$ ).

7. The actual calculation of the absolute invariants from the partial differential equations which they satisfy can be considerably abbreviated at a particular stage.

Dealing, for example, with the case in which $p=2$, so that we have derivatives of $\varphi$ of the second order, we have eight equations in the set (I). Six of these arise through the vanishing of $\xi_{20}, \xi_{11}, \xi_{02}, n_{20}, n_{11}, n_{02}$ in $\S_{4}$ : they are

$$
\begin{gathered}
2 a \frac{\partial f}{\partial a_{10}}+b \frac{\partial f}{\partial b_{10}}+\varphi_{10} \frac{\partial f}{\partial \varphi_{20}}=0 \\
2 a \frac{\partial f}{\partial a_{01}}+a \frac{\partial f}{\partial h_{10}}+b \frac{\partial f}{\partial b_{01}}+2 b \frac{\partial f}{\partial b_{10}}+\varphi_{10} \frac{\partial f}{\partial \varphi_{11}}=0 \\
a \frac{\partial f}{\partial h_{01}}+2 b \frac{\partial f}{\partial b_{01}}+\varphi_{10} \frac{\partial f}{\partial \varphi_{02}}=0 \\
2 b \frac{\partial f}{\partial a_{10}}+b \frac{\partial f}{\partial h_{10}}+\varphi_{01} \frac{\partial f}{\partial \varphi_{20}}=0 \\
2 b \frac{\partial f}{\partial a_{01}}+b \frac{\partial f}{\partial b_{10}}+b \frac{\partial f}{\partial b_{01}}+2 b \frac{\partial f}{\partial b_{10}}+\varphi_{01} \frac{\partial f}{\partial \varphi_{11}}=0 \\
b \frac{\partial f}{\partial b_{01}}+2 b \frac{\partial f}{\partial b_{01}}+\varphi_{01} \frac{\partial f}{\partial \varphi_{02}}=0 .
\end{gathered}
$$

These six equations are satisfied by

where

$$
f=a, b, b, \quad \varphi_{01}, \varphi_{\mathrm{ro}}, \quad A, H, B,
$$

$$
\begin{aligned}
& A=2 J^{2} \varphi_{20}-\left[b a_{10}-b\left(2 b_{10}-a_{\mathrm{O} 1}\right)\right] \varphi_{10}-\left[a\left(2 b_{10}-a_{01}\right)-b a_{10}\right] \varphi_{01} \\
& H=2 J^{2} \varphi_{11}-\left(b a_{01}-b b_{10}\right) \varphi_{10}-\left(a b_{10}-b a_{01}\right) \varphi_{01}, \\
& B=2 J^{2} \varphi_{02}-\left[b\left(2 b_{01}-b_{10}\right)-b b_{01}\right] \varphi_{10}-\left[a b_{01}-b\left(2 b_{01}-b_{10}\right)\right] \varphi_{01}
\end{aligned}
$$

*) See the two memoirs quoted at the beginning of this Note. 
any function of these eight quantities will satisfy the foregoing six equations. We require such combinations of them as will satisfy the remaining equations.

The two remaining equations in (I) arise out of the coefficients of $\xi_{01}$ and $\eta_{10}$ : they are respectively

$$
\begin{gathered}
\Delta_{1} f=a_{10} \frac{\partial f}{\partial a_{01}}+a_{10} \frac{\partial f}{\partial b_{10}}+\left(a_{01}+b_{10}\right) \frac{\partial f}{\partial b_{01}}+a \frac{\partial f}{\partial b}+2 b_{10} \frac{\partial f}{\partial b_{10}}+2 b \frac{\partial f}{\partial b} \\
+\left(b_{10}+2 b_{01}\right) \frac{\partial f}{\partial b_{01}}+\varphi_{20} \frac{\partial f}{\partial \varphi_{11}}+2 \varphi_{11} \frac{\partial f}{\partial \varphi_{02}}+\varphi_{10} \frac{\partial f}{\partial \varphi_{01}}=0, \\
s_{2} f=\left(a_{01}+2 b_{10}\right) \frac{\partial f}{\partial a_{10}}+2 b_{01} \frac{\partial f}{\partial a_{01}}+2 b \frac{\partial f}{\partial a}+\left(b_{01}+b_{10}\right) \frac{\partial f}{\partial b_{10}}+b_{01} \frac{\partial f}{\partial b_{01}}+b \frac{\partial f}{\partial b} \\
+b_{01} \frac{\partial f}{\partial b_{10}}+2 \varphi_{11} \frac{\partial f}{\partial \varphi_{20}}+\varphi_{02} \frac{\partial f}{\partial \varphi_{11}}+\varphi_{01} \frac{\partial f}{\partial \varphi_{10}}=0 .
\end{gathered}
$$

Now $f$ is to be a function of $a, b, b, \varphi_{01}, \varphi_{10}, A, H, B$ : hence, as

$$
\begin{aligned}
\Delta_{1} a & =0, & \Delta_{2} a & =2 b, \\
\Delta_{1} b & =a, & \Delta_{2} b & =b, \\
\Delta_{1} b & =2 b, & \Delta_{2} b & =0, \\
\Delta_{1} \varphi_{01} & =\varphi_{10}, & \Delta_{2} \varphi_{01} & =0, \\
\Delta_{1} \varphi_{10} & =0, & \Delta_{2} \varphi_{10} & =\varphi_{01}, \\
\Delta_{1} A & =0, & \Delta_{2} A & =2 H, \\
\Delta_{1} H & =A, & \Delta_{2} H & =B, \\
\Delta_{1} B & =2 H, & \Delta_{2} B & =0,
\end{aligned}
$$

the two equations become

$$
\begin{aligned}
& a \frac{\partial f}{\partial b}+2 b \frac{\partial f}{\partial b}+A \frac{\partial f}{\partial H}+2 H \frac{\partial f}{\partial B}+\varphi_{10} \frac{\partial f}{\partial \varphi_{01}}=0 \\
& b \frac{\partial f}{\partial b}+2 b \frac{\partial f}{\partial a}+B \frac{\partial f}{\partial H}+2 H \frac{\partial f}{\partial A}+\varphi_{0_{1}} \frac{\partial f}{\partial \varphi_{10}}=0
\end{aligned}
$$

and equation (II), similarly transformed, becomes

$$
2 a \frac{\partial f}{\partial a}-2 b \frac{\partial f}{\partial b}+2 A \frac{\partial f}{\partial A}-2 B \frac{\partial f}{\partial B}+\varphi_{\mathrm{Io}} \frac{\partial f}{\partial \varphi_{\mathrm{Io}}}-\varphi_{\mathrm{or}} \frac{\partial f}{\partial \varphi_{\mathrm{or}}}=0 .
$$

These three equations are characteristic of the invariants and covariants of the two binary forms

$$
\left(a, b, b \gamma \varphi_{01},-\varphi_{10}\right)^{2}, \quad\left(A, H, B \gamma \varphi_{01},-\varphi_{10}\right)^{2}:
$$

and therefore the required solutions of the equations are provided by any algebraically complete aggregate of those invariants and covariants. Such an aggregate can be taken in the form: 


$$
\begin{aligned}
& J^{2}=a b-b^{2}, \\
& I_{1}=\left(a, b, b \gamma \varphi_{01},-\varphi_{10}\right)^{2}, \\
& I_{2}=\left(A, H, B \gamma \varphi_{01},-\varphi_{10}\right)^{2}, \\
& I_{3}=\left(a H-b A, a B-b A, b B-b H \gamma \varphi_{01},-\varphi_{10}\right)^{2}, \\
& I_{4}=a B-2 b H+b A .
\end{aligned}
$$

Another invariant is given by $A B-H^{2}$ : but as

$$
I_{1} I_{2} I_{4}-I_{3}^{2}-J^{2} I_{2}^{2}=I_{1}^{2}\left(A B-H^{2}\right)
$$

it is expressible in terms of the others. It is better, for the sake of geometrical interpretation, to select invariants and covariants which do not involve $A, H, B$ to a degree higher than the first.

8. The equation (III) for the determination of $\mu$ must be similarly modified: it becomes

$$
\begin{gathered}
2\left(a \frac{\partial f}{\partial a}+b \frac{\partial f}{\partial b}+b \frac{\partial f}{\partial b}\right)+6\left(A \frac{\partial f}{\partial A}+H \frac{\partial f}{\partial H}+B \frac{\partial f}{\partial B}\right) \\
+\psi_{01} \frac{\partial f}{\partial \varphi_{01}}+\varphi_{10} \frac{\partial f}{\partial p_{10}}=2 \mu f .
\end{gathered}
$$

When we take the quantities in the succession

$$
J^{2}, I_{1}, I_{2}, I_{3}, I_{4}
$$

and substitute in this modified equation, we find the respective values of $\mu$ to be

$$
2, \quad 2,4,5,4 \text {. }
$$

Hence the absolute invariants are the four quantities

$$
\frac{I_{1}}{J^{2}}, \frac{I_{2}}{J^{4}}, \frac{I_{3}}{J^{5}}, \frac{I_{4}}{J^{4}}
$$

being the correct number, for $p=2$.

9. The geometrical significance of these invariants can be obtained in several ways: one of the simplest is the following. The invariants remain absolutely unaltered in value whatever changes be made in the variables: thus their values with $u$ and $v$ as variables are the same as their values with $x$ and $y$ as variables. When $x$ and $y$ are variables let

$$
f(x, y)=\text { constant }
$$

be the curve $\varphi(u, v)=$ constant, so that

$$
\varphi(u, v)=f(x, y) .
$$

For this selection of variables, we have

$$
\begin{aligned}
& a=\mathrm{I}, \quad b=\mathrm{o}, \quad b=\mathrm{I}, \quad J=\mathrm{I}, \\
& A=2 f_{x x}, \quad H=2 f_{x y}, \quad B=2 f_{y y} .
\end{aligned}
$$


Thus

$$
\begin{aligned}
& \frac{I_{1}}{J^{2}}=f_{x}^{2}+f_{y}^{2}, \\
& \frac{I_{2}}{J^{4}}=2\left(f_{x x} f_{y}^{2}-2 f_{x y} f_{x} f_{y}+f_{y y} f_{x}^{2}\right), \\
& \frac{I_{3}}{J^{5}}=2\left[\left(f_{x x}-f_{y y}\right) f_{x} f_{y}-\left(f_{x}^{2}-f_{y}^{2}\right) f_{x y}\right], \\
& \frac{I_{4}}{J^{4}}=2\left(f_{x x}+f_{y y}\right) .
\end{aligned}
$$

Let $l, m$ denote the direction-cosines of the tangent to the curve at any point; and let $l^{\prime}, m^{\prime}$ denote the direction-cosines of the normal to the curve at the same point.

Then

But

$$
\begin{aligned}
& \frac{l}{f_{y}}=\frac{m}{-f_{x}}=\frac{\mathrm{I}}{\left(f_{x}^{2}+f_{y}^{2}\right)^{\frac{1}{2}}}, \\
& \frac{l^{\prime}}{f_{x}}=\frac{m^{\prime}}{f_{y}}=\frac{\mathrm{I}}{\left(f_{x}^{2}+f_{y}^{2}\right)^{\frac{1}{2}}} .
\end{aligned}
$$

and therefore

$$
C=\frac{d \varphi}{d n}=l^{\prime} f_{x}+m^{\prime} f_{y}=\left(f_{x}^{2}+f_{y}^{2}\right)^{\frac{\mathrm{I}}{2}} ;
$$

Also

$$
\begin{aligned}
& l=\frac{\mathrm{I}}{C} f_{y}, \quad m=-\frac{\mathrm{I}}{C} f_{x}, \\
& l^{\prime}=\frac{\mathrm{I}}{C} f_{x}, \quad m^{\prime}=\frac{\mathrm{I}}{C} f_{y} .
\end{aligned}
$$

$$
\begin{aligned}
& \frac{d C}{d s}=\left(l \frac{d}{d x}+m \frac{d}{d y}\right)\left(f_{x}^{2}+f_{y}^{2}\right)^{\frac{1}{2}}=\frac{\mathrm{I}}{C^{2}}\left[\left(f_{x x}-f_{y y}\right) f_{x} f_{y}-\left(f_{x}^{2}-f_{y}^{2}\right) f_{x y}\right], \\
& \frac{d C}{d n}=\left(l^{\prime} \frac{d}{d x}+m^{\prime} \frac{d}{d y}\right)\left(f_{x}^{2}+f_{y}^{2}\right)^{\frac{1}{2}}=\frac{\mathrm{I}}{C^{2}}\left[f_{x}^{2} f_{x x}+f_{x} f_{y} f_{x y}+f_{y}^{2} f_{y y}\right]
\end{aligned}
$$

and, as usual,

$$
\frac{C^{3}}{\rho}=f_{x x} f_{y}^{2}-2 f_{x y} f_{x} f_{y}+f_{y y} f_{x}^{2} .
$$

These results at once give the following geometrical interpretations:

$$
\begin{aligned}
& \frac{I_{1}}{J^{2}}=C^{2}, \\
& \frac{I_{2}}{J^{4}}=\frac{2}{\rho} C^{3}, \\
& \frac{I_{3}}{J^{s}}=2 C^{2} \frac{d C}{d s}, \\
& \frac{I_{4}}{J^{4}}=2 \frac{d C}{d n}+\frac{2}{\rho} C ;
\end{aligned}
$$


where

$$
C=\frac{d \varphi}{d n}
$$

the rate of expansion along the normal to the curve.

ro. Corresponding results up to the same order are similarly obtainable when two curves are given in the plane in a form

$$
\varphi(u, v)=\text { constant, } \quad \varphi^{\prime}(u, v)=\text { constant; }
$$

it is unnecessary to consider the case of three curves

$$
\varphi(u, v)=\text { constant }, \quad \varphi^{\prime}(u, v)=\text { constant }, \quad \varphi^{\prime \prime}(u, v)=\text { constant, }
$$

for an identical relation connects $\varphi, \varphi^{\prime}, \varphi^{\prime \prime}$. It will be sufficient to state the results for two curves. Let

$$
\begin{aligned}
& A^{\prime}=2 J^{2} \varphi_{10}^{\prime}-\left[b a_{10}-b\left(2 b_{10}-a_{01}\right)\right] \varphi_{10}^{\prime}-\left[a\left(2 b_{10}-a_{01}\right)-b a_{10}\right] \varphi_{01}^{\prime}, \\
& H^{\prime}=2 J^{2} \varphi_{11}^{\prime}-\left(b a_{01}-b b_{10}\right) \varphi_{10}^{\prime}-\left(a b_{10}-b a_{0:}\right) \varphi_{01}^{\prime}, \\
& B^{\prime}=2 J^{2} \varphi_{02}^{\prime}-\left[b\left(2 b_{01}-b_{10}\right)-b b_{01}\right] \varphi_{10}^{\prime}-\left[a b_{01}-b\left(2 b_{01}-b_{10}\right)\right] \varphi_{01}^{\prime} ;
\end{aligned}
$$

and let

$$
\begin{aligned}
& I_{2}^{\prime}=\left(A^{\prime}, H^{\prime}, B^{\prime} \chi \varphi_{01}^{\prime},-\varphi_{10}^{\prime}\right)^{2}, \\
& I_{3}^{\prime}=\left(a H^{\prime}-b A^{\prime}, a B^{\prime}-b A^{\prime}, b B^{\prime}-b H^{\prime} \gamma\left(\varphi_{01}^{\prime},-\varphi_{10}^{\prime}\right)^{2},\right. \\
& I_{4}^{\prime}=a B^{\prime}-2 b H^{\prime}+b A^{\prime}, \\
& K=\left(\varphi_{10}^{\prime}, \varphi_{01}^{\prime} \gamma \varphi_{01},-\varphi_{10}\right), \\
& F=\left(a, b, b \chi \varphi_{01}^{\prime},-\varphi_{10}^{\prime} \chi \varphi_{01},-\varphi_{10}\right) .
\end{aligned}
$$

Then the absolute invariants for the two curves can be represented by

$$
\begin{aligned}
& \frac{K}{J}, \\
& \frac{I^{\prime}}{J^{2}}, \quad \frac{F}{J^{2}}, \\
& \frac{I_{2}}{J^{4}}, \quad \frac{I_{4}}{J^{4}}, \quad \frac{I_{2}^{\prime}}{J^{4}}, \quad \frac{I_{4}^{\prime}}{J^{4}}, \\
& \frac{I_{3}}{J^{5}}, \quad \frac{I_{3}^{\prime}}{J^{5}} ;
\end{aligned}
$$

or they can be represented by any similar (and equivalent) aggregate. Thus $F$ could be replaced by $I_{\mathrm{t}}^{\prime}$, where

owing to the relation

$$
I_{\mathrm{r}}^{\prime}=\left(a, b, b \gamma \varphi_{01}^{\prime},-\varphi_{10}^{\prime}\right)^{2}
$$

or it could be replaced by

$$
I_{\mathrm{I}} I_{\mathrm{I}}^{\prime}-F^{2}=J^{2} K^{2}
$$

$$
\Gamma=\left(A, H, B \gamma \varphi_{10},-\varphi_{10} \chi \varphi_{01}^{\prime},-\varphi_{10}^{\prime}\right)
$$


owing to the relation

and so for other possibilities.

$$
I_{1} \Gamma+I_{3} K=I_{2} F
$$

II. As regards the geometrical interpretation, we retain the former notation for quantities connected with the curve $\phi(u, v)=$ constant; and we use corresponding symbols for quantities connected with the curve $p^{\prime}(u, v)=$ constant. Let $d s^{\prime}$ and $d n^{\prime}$ denote elements of length in the direction of the tangent and the normal of the latter curve respectively; let

$$
C^{\prime}=\frac{d \varphi^{\prime}}{d n^{\prime}}
$$

and let $\rho^{\prime}$ denote the radius of curvature of $\phi^{\prime}=$ constant at the point; and let $\omega$ denote the angle at which the curves intersect. Then

$$
\frac{I_{x}}{J^{2}}, \quad \frac{I_{2}}{J^{4}}, \quad \frac{I_{4}}{J^{4}}, \quad \frac{I_{3}}{J^{3}}
$$

have their former values; also

$$
\begin{aligned}
& \frac{I_{2}^{\prime}}{J^{4}}=\frac{2}{\rho^{\prime}} C^{\prime 3}, \\
& \frac{I_{3}^{\prime}}{J^{s}}=2 C^{\prime 2} d C^{\prime} d s^{\prime}, \\
& \frac{I_{4}^{\prime}}{J^{4}}=2 \frac{d C^{\prime}}{d n^{\prime}}+\frac{2}{\rho^{\prime}} C^{\prime}, \\
& \frac{K}{J}=C C^{\prime} \sin \omega, \\
& \frac{F}{J^{2}}=C C^{\prime} \cos \omega .
\end{aligned}
$$

In terms of these nine absolute invariants, all other invariants, of the same order and belonging to the two curves, can be expressed.

Cambridge (Engl.), July 16, 1905.

A. R. FORSYTH. 